\title{
Single-nucleotide polymorphism arrays and unexpected consanguinity: considerations for clinicians when returning results to families
}

\author{
Fernanda Delgado, BS ${ }^{1,2}$, Holly K. Tabor, $\mathrm{PhD}^{2-4}$, Penny M. Chow, MS³, Jessie H. Conta, MS, \\ Kenneth W. Feldman, MD'3 , Karen D. Tsuchiya, MD³ and Anita E. Beck, MD, PhD ${ }^{1-3}$
}

\begin{abstract}
Purpose: The broad use of single-nucleotide polymorphism microarrays has increased identification of unexpected consanguinity. Therefore, guidelines to address reporting of consanguinity have been published for clinical laboratories. Because no such guidelines for clinicians exist, we describe a case and present recommendations for clinicians to disclose unexpected consanguinity to families.

Methods: In a boy with multiple endocrine abnormalities and structural birth defects, single-nucleotide polymorphism array analysis revealed $\sim 23 \%$ autosomal homozygosity suggestive of a first-degree parental relationship. We assembled an interdisciplinary health-care team, planned the most appropriate way to discuss results of the single-nucleotide polymorphism array with the adult mother, including the possibility of multiple autosomal recessive disorders in her child, and finally met with her as a team.
\end{abstract}

Results: From these discussions, we developed four major considerations for clinicians returning results of unexpected consanguinity, all guided by the child's best interests: (i) ethical and legal obligations for reporting possible abuse, (ii) preservation of the clinical relationship, (iii) attention to justice and psychosocial challenges, and (iv) utilization of the single-nucleotide polymorphism array results to guide further testing.

Conclusion: As single-nucleotide polymorphism arrays become a common clinical diagnostic tool, clinicians can use this framework to return results of unexpected consanguinity to families in a supportive and productive manner.

Genet Med advance online publication 18 September 2014

Key Words: clinical ethics; consanguinity; DNA arrays; genetic counseling; incest
Approximately $10 \%$ of the global population is related as second cousins or closer. ${ }^{1}$ Not surprisingly, long contiguous stretches of homozygosity have been found in genomes across global populations. ${ }^{2}$ Reasons for consanguineous unions encompass a variety of cultural, political, religious, and geographic issues. ${ }^{1,3,4}$ However, levels of malformations and significant medical defects are somewhat higher among the offspring of first cousins $(4.4 \%)$ as compared with those of unrelated parents and parents who are at least second cousins (3.6\%). ${ }^{5}$ In some countries, including the United States, some close marriages are banned by law. ${ }^{1,6}$ In addition, evidence of close consanguinity often raises questions about the possibility of unreported or undetected abuse and/or incest.

Taking a family history to construct an accurate pedigree, including asking about the possible relatedness of family members, has traditionally been an integral part of a medical genetics evaluation. ${ }^{7}$ When describing family relationships, patients may reveal known consanguinity that is clinically relevant. The introduction of single-nucleotide polymorphism (SNP) microarray testing has noticeably increased the identification of unexpected and/or unreported consanguinity. ${ }^{8-11}$ SNP arrays, like array comparative genomic hybridization, are often used in diagnostic testing for individuals with birth defects, intellectual disabilities, and/or autism spectrum disorders to reveal genomic copy-number variants such as deletions and duplications. Unlike array comparative genomic hybridization, SNP arrays can also reveal long contiguous stretches of homozygosity. These stretches of homozygosity can represent consanguinity, shared ancestry, or isodisomic uniparental disomy, ${ }^{12}$ each of which is associated with an increased incidence of autosomal recessive disorders.

The American College of Medical Genetics and Genomics recently published guidelines to assist laboratories in documenting for the ordering physician suspected consanguinity as an incidental finding of genomic testing, ${ }^{13}$ in response to the variability in laboratory reporting practices. ${ }^{14}$ However, no formal guidelines currently exist for ordering clinicians to disclose findings of unexpected consanguinity to families while considering potential legal reporting obligations. ${ }^{15-17}$ In this article we describe a recent illustrative case of unexpected consanguinity and propose practical and ethical considerations for ordering clinicians when returning results of unexpected consanguinity in the clinical setting.

\footnotetext{
${ }^{1}$ University of Washington School of Medicine, Seattle, Washington, USA; ${ }^{2}$ Division of Genetic Medicine, Department of Pediatrics, University of Washington, Seattle, Washington, USA; ${ }^{3}$ Seattle Children's Hospital, Seattle, Washington, USA; ${ }^{4}$ Treuman Katz Center for Pediatric Bioethics, Seattle Children's Research Institute, Seattle, Washington, USA. Correspondence: Anita E. Beck (aebeck@uw.edu) 


\section{CLINICAL REPORT}

We present the case of an 8-week-old male born vaginally at term to a 24 -year-old gravida 1 , para 1 mother and a 22 -yearold father. His mother took only prenatal vitamins and denied alcohol, tobacco, and recreational drug use during the pregnancy. Prenatal ultrasounds were normal and prenatal genetic diagnostic studies were not done.

The patient was found to have both endocrine and structural abnormalities, including congenital primary hypothyroidism, hypoglycemia with concurrent hyperinsulinemia, deficiency in growth hormone and cortisol, prolonged direct and indirect hyperbilirubinemia, hypertrichosis, and anemia. An echocardiogram showed a patent foramen ovale and mild left pulmonary artery branch stenosis. Brain magnetic resonance imaging suggested mild generalized volume loss with slight thinning of the body of the corpus callosum. The pituitary gland and stalk were normal in size, position, and signal without mass effect on the optic chiasm. The patient was hypotonic centrally, with moderate dysphagia leading to episodes of aspiration. Standardized neurodevelopmental testing confirmed significant global developmental delay by 6 months of age.

A three-generation pedigree showed that all the members of this extended Mexican family were healthy. The mother denied consanguinity and reported that the father of the child, who was no longer involved with the mother or child, was from a separate region of Mexico.

After clinical evaluation, specialists in pediatric genetics and endocrinology were unable to reach a unifying genetic diagnosis. Following pretest counseling, an Affymetrix SNP array was ordered.

\section{RESULTS}

The SNP array analysis did not identify any clinically significant deletions or duplications. It did, however, identify 23\% autosomal homozygosity across multiple chromosomes (affecting a total of $\sim 664 \mathrm{Mb}$, blocks $\geq 3 \mathrm{Mb}$ ). This level of homozygosity is consistent with a close parental relationship or more distant relatedness in an isolated population (Figure 1a).

The interdisciplinary health-care team, consisting of a clinical geneticist, genetic counselor, social worker, medical Spanish interpreter, and a patient advocate, discussed the results of the SNP array with the mother and the implications for the child's health. The mother denied consanguinity and/or sexual abuse during this and several other visits. We left open the opportunity for the mother to disclose consanguinity in the future, should she need additional psychosocial resources.

In accordance with our hospital policies, we report unexpected consanguinity to the child-protection team when the safety of the mother (if a minor at conception or intellectually impaired) and/or the safety of the child (if abuse is suspected) are at risk. We decided not to report this situation to the hospital's child-protection team for several reasons. First, the mother was an adult and not intellectually impaired. Second, she denied abuse of herself and her son. Third, the father of the patient was no longer involved. Finally, we wanted to maintain a

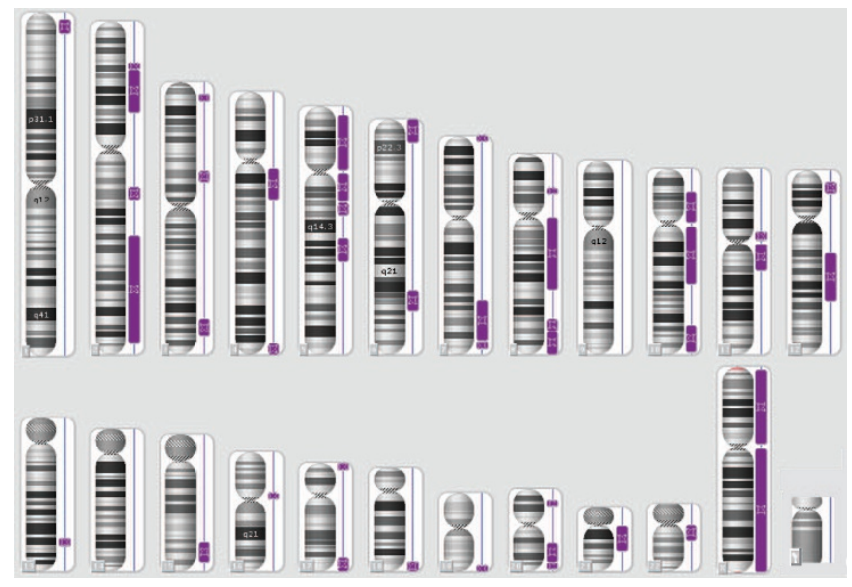

b

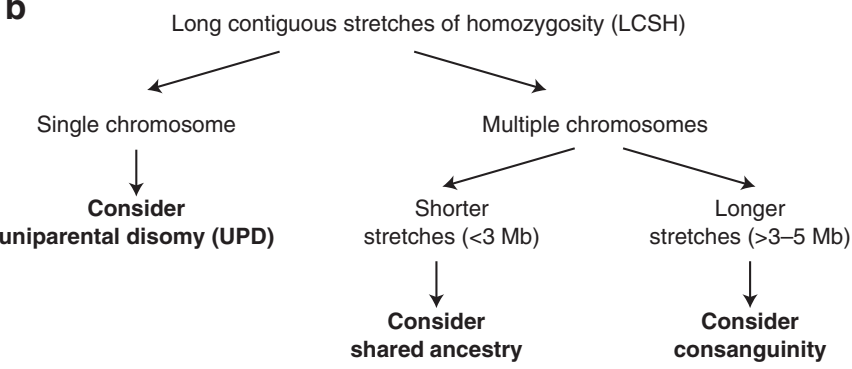

Figure 1 Determining whether homozygous regions may represent consanguinity. (a) A visual representation of all of the regions of homozygosity $\geq 3 \mathrm{Mb}$ (purple blocks) in the proband. These long stretches of homozygosity are located on multiple chromosomes. Note that the $X$ chromosome also appears to be homozygous but in fact is present in the normal hemizygous state because this individual is male. (b) A decision tree to help determine whether stretches of homozygosity represent uniparental disomy, shared ancestry, or consanguinity. Caution should be exercised, however, because a result of high homozygosity alone is insufficient to claim consanguinity. ${ }^{9}$

a collaborative clinical relationship with the mother for the optimal care of the child. From a medical standpoint, the SNP array results suggested that this patient was likely affected by one or more autosomal recessive disorders within the homozygous regions. We then investigated these regions using clinical exome sequencing and found a homozygous mutation in a gene for primary cortisol deficiency, explaining at least part of the patient's phenotype.

\section{DISCUSSION}

Long contiguous stretches of homozygosity on SNP arrays can represent isodisomic uniparental disomy, shared ancestry, or consanguinity, depending on the size and location of the homozygous regions (Figure $1 \mathrm{~b}$ ). Genomic homozygosity can help physicians identify DNA regions containing genes for autosomal recessive conditions but may also reveal unexpected consanguinity. ${ }^{8}$ When unexpected, consanguinity can be difficult to discuss with families because of both potential adverse health outcomes for the child ${ }^{4,5,11,18}$ and legal implications for the parent(s). ${ }^{6,15,17}$ 
As this case demonstrates, pretest counseling before ordering a SNP array should include an explanation that consanguinity and potential relatedness may be identified. Providers can explain to parents that such a finding of consanguinity could be beneficial for making a diagnosis for their child. This process of pretest counseling paves the way for future discussions if unexpected consanguinity is discovered. This process also allows families to be fully informed when consenting to or declining testing. If families decline, providers should explore and address the parents' specific concerns. Such a discussion may prompt a parent to make comments that lead a provider to suspect abuse.

Based on our experience with this case, we developed recommendations for the review of cases of unexpected consanguinity identified through SNP arrays, including considerations for how to report the results to a family and whether the child-protection team should be notified. We recommend that, when possible, the issues raised by the detection of unexpected consanguinity be addressed through the formation of an interdisciplinary care team. Our team comprised a medical genetics physician, a medical genetics counselor, a social worker, a medical Spanish interpreter, and a patient advocate, with input from a bioethicist. The specific roles of each team member are described in Figure 2a. This interdisciplinary care team model allows for the effective consideration of medical, ethical, and reporting issues in the specific context of a case, and it may be useful in other clinical environments when the expertise is available. If the family feels overwhelmed by the team approach, one trusted member of the medical team may serve as the primary contact between the family and the interdisciplinary care team.

We identified four major considerations for clinicians when returning results of unexpected consanguinity, all guided by the child's best interests: (i) ethical and legal obligations for reporting possible abuse, (ii) preservation of the clinical relationship, (iii) attention to justice and psychosocial challenges, and (iv) utilization of the SNP array results to guide further testing.

First, the team should consider potential identification of, and reporting obligations stemming from, possible abuse. If either parent of the affected child is a minor and/or intellectually impaired, the treating physician may have ethical and legal obligations for reporting possible incest/abuse. Because

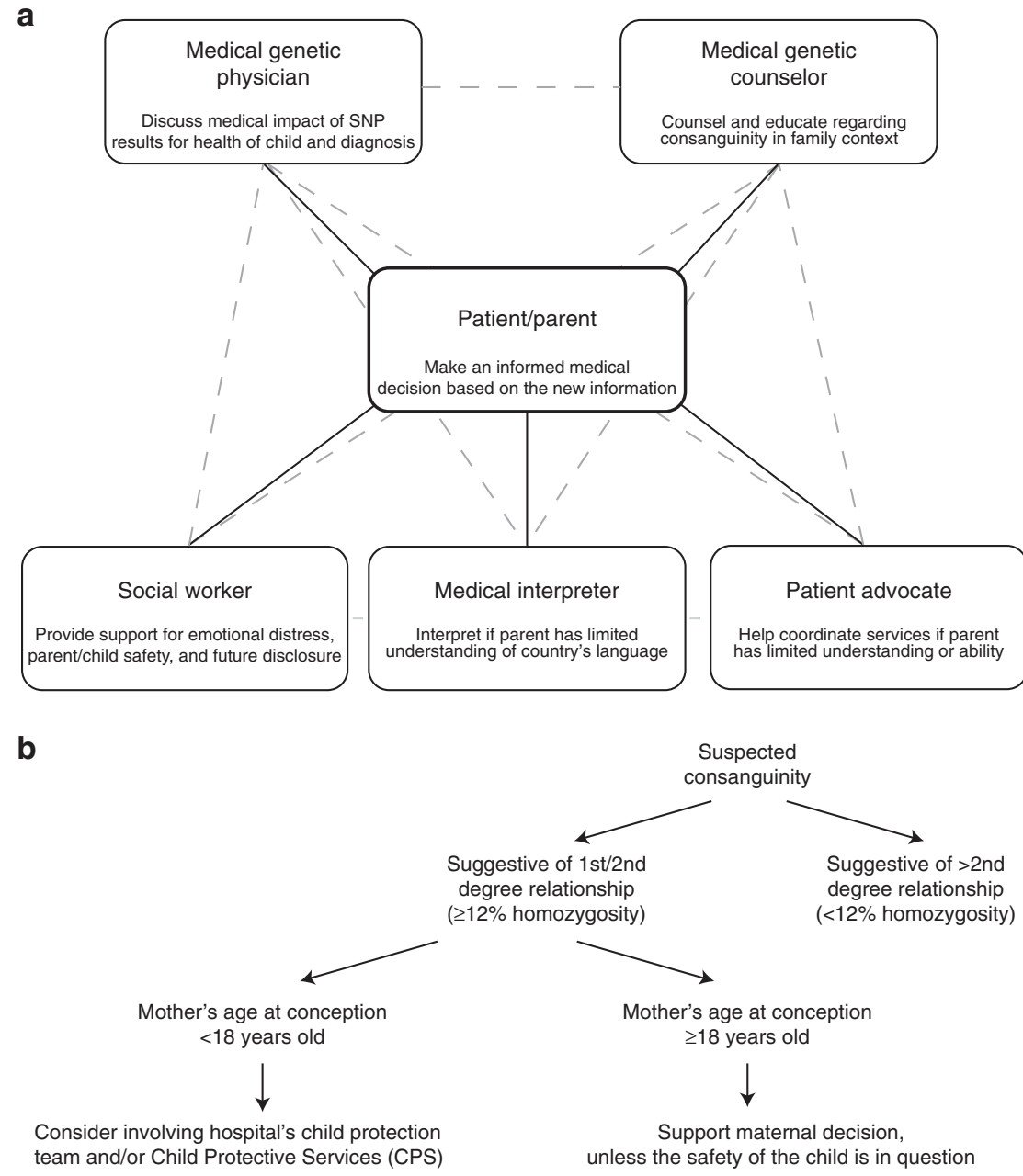

Figure 2 Suggested path for the clinician to disclose consanguinity to the patient or family. (a) Roles of the interdisciplinary team members who work with the patient/parent to support the best interests of the child. (b) Once consanguinity is suspected (from Figure 1b), a decision process is necessary to decide whether the situation should be reported to a child-protection group. 
consanguinity laws vary by state, providers need to be aware of their local applicable statutes. In the case of unexpected consanguinity involving a minor parent, the hospital's child abuse protection team should be involved to assess risk to this parent and/or the child (Figure 2b). Subsequent involvement with child protective services and law enforcement may be necessary.

If both parents are legal adults and not intellectually impaired, it is still possible that abuse has occurred and might be ongoing. If a family member discloses abuse in discussions before or after testing, the team should provide support from a social worker while respecting the abused adult's autonomy to decide whether to report such abuse, unless the safety of the child is in question. If the family denies abuse, then a few scenarios may be possible: (i) a parent has been sexually abused and is not ready to disclose the abuse at this time, (ii) the parents know that they are closely related but may not choose to disclose their consanguineous union to the providers because of shame or fear of potential societal or legal implications, ${ }^{19}$ or (iii) the parents do not know or understand that they are closely related. If the parents are consenting adults and disclose a consanguineous relationship such as a first cousin relationship, legal publications have reported that the provider may not be required to break provider-patient confidentiality and report this union, even if it is unlawful. 15,20

Second, because any of these scenarios can cause emotional distress and repercussions for the family, the clinician must undertake the discussion with the family in a cautious and thoughtful manner. Sensitivity is critical to the preservation of the clinical relationship with the family and is also in the best interests of the child by allowing ongoing care and communication. In particular, care must be taken to preserve the dignity of the individuals involved, especially if the family considers consanguinity to be shameful. ${ }^{7}$ In our case, the interdisciplinary care team decided to proceed without a strong confrontation with the mother. At the same time, we continued to offer support in the form of a social worker and patient advocate should she decide to disclose possible abuse in the future. In this way we could work to maintain the mother's trust while continuing to evaluate and treat the child.

Third, many patients and families vary in their education, literacy, language fluency, and economic status. To ensure equitable treatment for all patients while returning complex results of genetic testing that suggest consanguinity, it is prudent to include a social worker, a medical interpreter (when appropriate), and a patient advocate on the care team (Figure 2a). Conversely, withholding the option to discuss SNP array results that suggest consanguinity from specific patients and/or families is paternalistic, may be a form of dishonesty, and should be avoided.

Fourth, once the results have been disclosed and discussed, the provider must determine how to use the SNP array results to continue the child's diagnostic evaluation. Given the SNP array results, the hypothesis may be that the child has one or more autosomal recessive disorders, and therefore genes that lie within the homozygous regions are possible candidate genes. If mutations in one or a few of these candidate genes could likely cause the child's disorder(s), then targeted sequencing of that gene(s) can be undertaken. If no obvious causative candidate gene is identified, then exome (or whole-genome) sequencing may be considered. Regardless of the next steps, ongoing diagnostic evaluation and treatment of the child, and the pursuit of the clinical best interest of the child, frequently require maintaining trust and communication with a family. Therefore, if the parents were both adults at the time of conception, and there is no suspicion or disclosure of abuse, it may not be beneficial to pressure the family to disclose the exact nature of the familial relationships because the SNP array results guide further diagnostic testing.

In conclusion, we used a clinical case to illustrate an interdisciplinary and practical approach for ordering providers to utilize when planning to disclose to families SNP array results suggestive of consanguinity. The child's best interest is paramount and is supported by maintaining ongoing trust and communication with the family while balancing legal reporting obligations.

\section{ACKNOWLEDGMENTS}

Our work was supported by a grant from the National Institutes of Health $(\mathrm{NIH}) /$ National Institute of Child Health and Human Development (5K23HD057331 to A.E.B.), a grant from the NIH/National Human Genome Research Institute (5ROOHG004316-0 to H.K.T.), and a grant from the American College of Medical Genetics Foundation Summer Scholars Program to F.D. The authors thank Fuki Hisama for mentoring support.

\section{DISCLOSURE}

The authors declare no conflict of interest.

\section{REFERENCES}

1. Bittles $A H$, Black ML. Evolution in health and medicine Sackler colloquium: consanguinity, human evolution, and complex diseases. Proc Natl Acad Sci USA 2010;107(suppl 1):1779-1786.

2. Kirin M, McQuillan R, Franklin CS, Campbell H, McKeigue PM, Wilson JF. Genomic runs of homozygosity record population history and consanguinity. PLoS One 2010;5:e13996.

3. Modell B, Darr A. Science and society: genetic counselling and customary consanguineous marriage. Nat Rev Genet 2002;3:225-229.

4. Hamamy H, Antonarakis SE, Cavalli-Sforza LL, et al. Consanguineous marriages, pearls and perils: Geneva International Consanguinity Workshop Report. Genet Med 2011;13:841-847.

5. Zlotogora J, Shalev SA. The consequences of consanguinity on the rates of malformations and major medical conditions at birth and in early childhood in inbred populations. Am J Med Genet A 2010;152A:2023-2028.

6. Bittles $A$. Consanguinity and its relevance to clinical genetics. Clin Genet 2001;60:89-98.

7. Bennett RL, Motulsky AG, Bittles A, et al. Genetic counseling and screening of consanguineous couples and their offspring: recommendations of the National Society of Genetic Counselors. J Genet Counsel 2002;11:97-119.

8. Sund KL, Zimmerman SL, Thomas C, et al. Regions of homozygosity identified by SNP microarray analysis aid in the diagnosis of autosomal recessive disease and incidentally detect parental blood relationships. Genet Med 2013;15:7078.

9. Helm BM, Langley K, Spangler B, Vergano S. Three clinical experiences with SNP array results consistent with parental incest: a narrative with lessons learned. $J$ Genet Couns 2014;23:489-495. 
10. Tarini BA, Konczal LL, Goldenberg AJ, Goldman EB, McCandless SE. The perils of SNP microarray testing: uncovering unexpected consanguinity. Pediatr Neurol 2013;49:50-53.

11. Fan $Y S$, Ouyang $X$, Peng J, et al. Frequent detection of parental consanguinity in children with developmental disorders by a combined CGH and SNP microarray. Mol Cytogenet 2013;6:38.

12. Kearney HM, Kearney JB, Conlin LK. Diagnostic implications of excessive homozygosity detected by SNP-based microarrays: consanguinity, uniparental disomy, and recessive single-gene mutations. Clin Lab Med 2011;31:595-613, ix.

13. Rehder CW, David KL, Hirsch B, Toriello HV, Wilson CM, Kearney HM. American College of Medical Genetics and Genomics: standards and guidelines for documenting suspected consanguinity as an incidental finding of genomic testing. Genet Med 2013;15:150-152.

14. Grote L, Myers M, Lovell A, Saal H, Lipscomb Sund K. Variability in laboratory reporting practices for regions of homozygosity indicating parental relatedness as identified by SNP microarray testing. Genet Med 2012;14:971-976.
15. McGuire AL, Wang MJ, Probst FJ. Currents in contemporary bioethics. Identifying consanguinity through routine genomic analysis: reporting requirements. J Law Med Ethics 2012;40:1040-1046.

16. Downing NR, Williams JK, Daack-Hirsch S, Driessnack M, Simon CM. Genetics specialists' perspectives on disclosure of genomic incidental findings in the clinical setting. Patient Educ Couns 2013;90:133-138.

17. Driessnack M, Daack-Hirsch S, Downing N, et al. The disclosure of incidental genomic findings: an "ethically important moment" in pediatric research and practice. J Community Genet 2013;4:435-444.

18. Shieh JT, Bittles $A H$, Hudgins L. Consanguinity and the risk of congenital heart disease. Am J Med Genet A 2012;158A:1236-1241.

19. Bennett RL, Hudgins L, Smith CO, Motulsky AG. Inconsistencies in genetic counseling and screening for consanguineous couples and their offspring: the need for practice guidelines. Genet Med 1999;1:286-292.

20. Goldman MJ, Gutheil TG. The misperceived duty to report patients' past crimes. Bull Am Acad Psychiatry Law 1994;22:407-410. 